\title{
USO DA TÉCNICA DE DIFRAÇÃO DE RAIOS $X$ CARACTERIZAÇÃO MICROESTRUTURAL DE BAUXITA POR DIFRAÇÃO DE RAIOS $X$
}

\section{USE OF X-RAY DIFFRACTION TECHNIQUE - MICROESTRUCTURAL CHARACTERIZATION OF BAUXITE BY DIFFRACTION OF X-RAYS}

\author{
M.Z. LOUZADA ${ }^{1}$, A. R. BIGANSOLLI ${ }^{1}$ e B. B. LIMA-KÜHN ${ }^{1}$ \\ ${ }^{1}$ Universidade Federal Rural do Rio de Janeiro, Departamento de Engenharia Química, \\ Brasil. \\ E-mail: maurozamp94@gmail.com
}

article info

Article history:

Received 6 July 2016

Accepted 3 January 2017

Available online 6 January 2017
PALAVRAS-CHAVE: Bauxita; Caracterização; Difração de Raios X. KEYWORDS: Bauxite; Characterization; X-Ray diffraction.

RESUMO: A bauxita é um minério de elevada importância industrial, sendo muito utilizada para a obtenção de alumínio metálico e seus compostos, bem como matéria-prima para produção de agentes coagulantes. $O$ Brasil, além de possuir grandes reservas, é também um dos maiores produtores do minério, ocupando lugar de destaque no cenário mundial. Apesar de ser frequentemente descrita como um minério de alumínio, a bauxita não é uma espécie mineral propriamente dita, mas um material heterogêneo formado de uma mistura de hidróxidos de alumínio hidratados contendo impurezas, sendo a Gibbsita, Boehmita e o Diásporo os seus principais constituintes. O objetivo deste trabalho é caracterizar uma amostra de bauxita utilizada na fabricação de sulfato de alumínio, através da Difração de Raios X (DRX), comparando o difratograma da amostra com padrões simulados no PowderCell. Os resultados obtidos mostram que a bauxita caracterizada é composta majoritariamente pela Gibbisita.

\begin{abstract}
Bauxite is an ore of high industrial importance, commonly used to extract metallic aluminium, its compounds, as well as raw material for the production of coagulants agents. Brazil, besides owning the largest reserves, it's also one of the biggest producers of that ore, occupying a prominent place on the international scene. Its formation occurs in tropical and subtropical regions due to the exposure of aluminosilicate rocks to weathering conditions. Despite being frequently described as an aluminium ore, bauxite is not exactly a mineral species, but is in fact a heterogenic material formed by a mixture of hydrated aluminium hydroxides containing impurities. The minerals Gibbsite, Boehmite and Diaspore are its main components. The purpose of this study is to characterize by X-Ray Diffraction (XRD) a sample of bauxite used in the production of aluminium sulfate, comparing diffratogram results with patterns simulated by PowderCell.
\end{abstract}

\section{INTRODUÇÃO}

A bauxita é um minério que possui elevada importância industrial para a obtenção do alumínio metálico e de seus compostos. O Brasil possui grandes reservas, especialmente nos estados do Pará e Minas Gerais, sendo também extraída em outras regiões como Maranhão, Goiás e São Paulo, ocupando lugar de destaque no cenário mundial. Segundo a U.S. 
Geological Survey, no ano de 2015, a produção de bauxita e de alumina instalada no Brasil foi da ordem de 33 milhões de toneladas de bauxita e 10 milhões de toneladas de alumina; e em 2016, os maiores produtores, em ordem decrescente, foram: Austrália, Brasil e China, com um total de 70\% da produção mundial (U.S. Geological Survey, 2017).

Esta é a principal fonte do alumínio metálico, que é processado a partir da alumina produzida através de seu hidróxido obtido pelo processo Bayer. Além de o hidróxido ser empregado na produção do alumínio, é também utilizado como matéria-prima de diversos setores da indústria, como para fabricação de materiais refratários, abrasivos, velas de ignição, cerâmicas e outros. Também tem aplicação direta nas indústrias de papel, tintas, vidros, produtos farmacêuticos, cremes dentais e retardantes de chamas (EVANS, 1995) e na manufatura de produtos químicos, particularmente de zeólitas e de sulfato de alumínio "livre de ferro". Outros usos importantes do hidróxido de alumínio são as produções de fluoreto de alumínio, nitrato de alumínio, policloreto de alumínio, poli-sulfatossilicato de alumínio, aluminato de sódio, catalisadores e pigmentos à base de titânio (EVANS, 1995; GREENWOOD, 1997).

A Bauxita se forma em regiões tropicais e subtropicais por ação do intemperismo sobre rochas aluminossilicáticas, sendo frequentemente descrita como um minério do alumínio; no entanto, esta não é uma espécie mineral propriamente dita, mas um material heterogêneo formado de uma mistura de hidróxidos de alumínio hidratados $\left(\left[\mathrm{AlO}_{\mathrm{x}}(\mathrm{OH})_{3-2 \mathrm{x}}\right], 0<\mathrm{x}<1\right)$ contendo óxidos de ferro, dióxido de titânio, silicatos de alumínio, várias misturas de sílica e outras impurezas em menores quantidades. Ela ocorre principalmente como "monohidratada" $\mathrm{AlOOH}$ (boehmita e diásporo) e "tri-hidratada" $\mathrm{Al}(\mathrm{OH})_{3}$ (gibbsita), sendo que as proporções das três formas variam dependendo da localização geográfica do minério. As bauxitas mais ricas em boehmita são encontradas em depósitos europeus (França e Grécia), enquanto que aquelas ricas em diásporo, na China, Hungria e Romênia. Em áreas de clima tropical como Jamaica, Brasil, Austrália, Guiné, Guiana, Suriname e Índia ocorrem as bauxitas geologicamente mais novas, que apresentam maior interesse comercial, ricas em gibbisita presente (EVANS, 1995; GREENWOOD, 1997).

A Gibbisita possui estrutura cristalina pseudohexagonal tubular, estrutura proposta pela primeira vez por Pauling (Pauling, 1930) e confirmada por Megaw (Megaw, 1934), sendo refinada pelo método dos mínimos quadrados por Saalfeld e Wedde (Saalfeld, 1973) através de medidas utilizando radiações de $\mathrm{CuK} \alpha$, onde os seguintes valores de parâmetros de rede foram obtidos: $\mathrm{a}=8,684 \pm 0,001 \AA, \mathrm{b}=5,078 \pm 0,001 \AA, \mathrm{c}=9,736 \pm 0,002 \AA$ e $\beta=94,54 \pm$ $0,01^{\circ}$.

O objetivo do presente trabalho é investigar por Difração de Raios X as fases presentes na bauxita, utilizada na fabricação de coagulantes, extraída da região de Lavrinhas - SP.

\section{MATERIAL E MÉTODOS}

A Bauxita, minimamente processada (seca e moída), utilizada no processo de fabricação do coagulante Sulfato de Alumínio foi caracterizada por Difração de Raios X (DRX). As medidas desta caracterização foram efetuadas à temperatura ambiente, utilizando radiação de $\mathrm{CuK} \alpha$, tensão de $30 \mathrm{kV}$, corrente de $15 \mathrm{~mA}$, em um difratômetro Rigaku modelo 
Miniflex II. As condições de medição foram de $20^{\circ}<2 \theta<90^{\circ}$, passo angular de $0,05^{\circ}$ e tempo de contagem de $30 \mathrm{~s}$. As fases presentes na amostra foram identificadas por comparação do difratograma experimental com padrões simulados através do programa PowderCell (KRAUS, 1999) a partir dos dados cristalográficos publicados por Pearson e Calvert (PEARSON, 1991).

\section{RESULTADOS E DISCUSSÕES}

Neste trabalho, as fases presentes na bauxita foram identificadas por comparação do difratograma experimental com os padrões simulados através do software PowderCell. Com a realização da análise da amostra de bauxita por DRX, foi obtido um registro gráfico dos sinais originados nos detectores eletrônicos de radiação pelas reflexões: o difratograma experimental (Figura 1), contendo os picos correspondentes aos planos (h, k, l) de difração da radiação pela estrutura do material estudado. Além deste modo de apresentação de resultados, também se obteve um arquivo numérico contendo os valores das intensidades relativas (cps) para cada posição $2 \theta$ que foram inseridos no software PowderCell para representar o difratograma da amostra. Sendo assim, nas Figuras de 2 a 10 são apresentadas as sobreposições do difratograma da amostra com os padrões simulados.

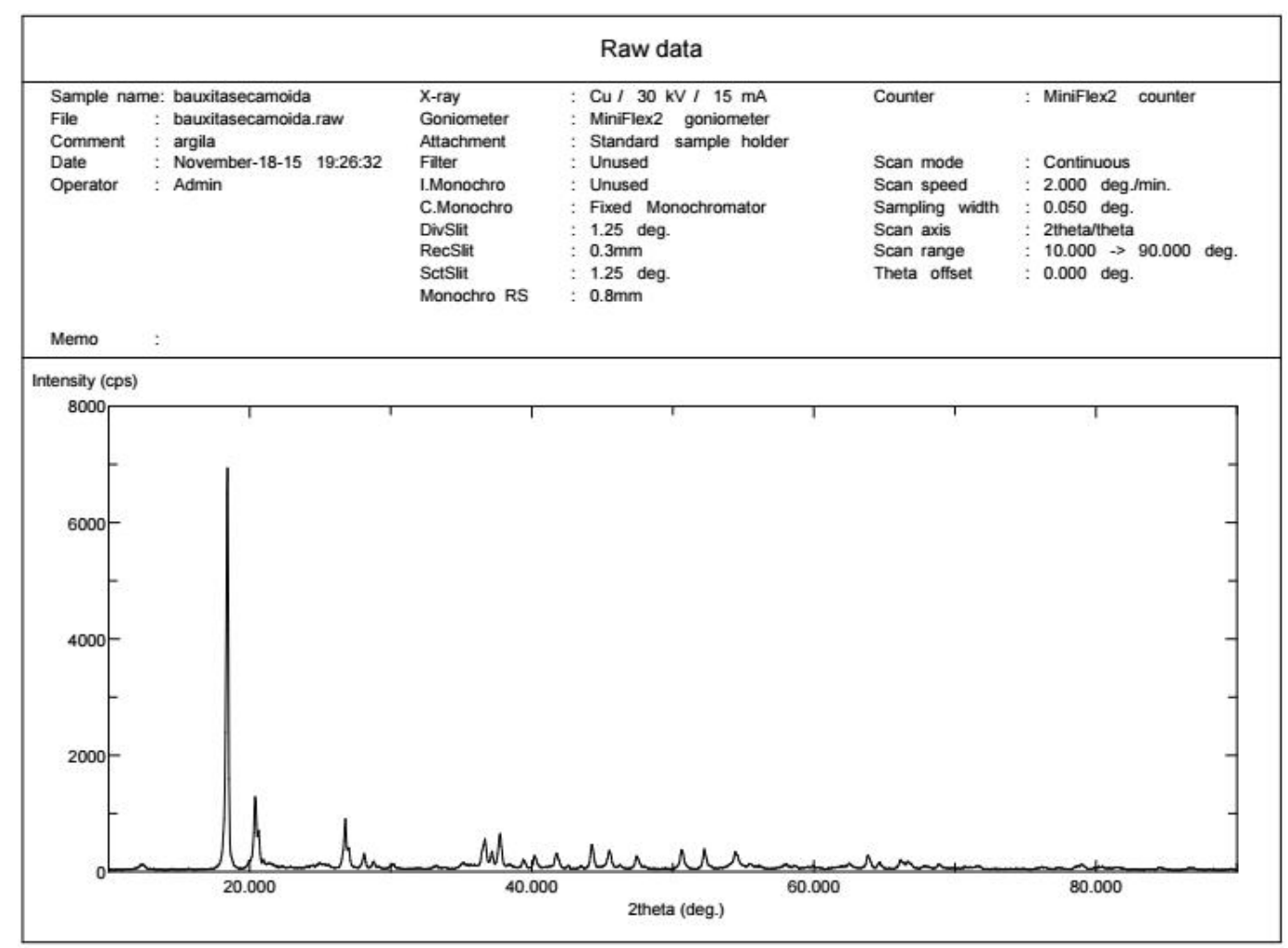

Figura 1 - Difratograma experimental da amostra de bauxita. 
Primeiramente, o difratograma experimental da amostra de bauxita foi sobreposto com o padrão simulado da Gibbsita e seus polimorfos Diásporo e Boehmita. Em seguida, o difratograma foi sobreposto às fases constituídas por óxidos de ferro como: hematita e magnetita, e às fases constituídas por aluminossilicatos, sílicas e óxidos de titânio representados respectivamente pelos padrões de Caulinita, Quartzo, Rutilo e Anatásio.

Na Figura 2 observa-se que o difratograma da amostra possui picos coincidentes com os picos do padrão simulado para a fase Gibbisita. Já nas Figuras 3 e 4, onde são apresentados os padrões das fases Diásporo e Boehmita não são observados a sobreposição dos picos da amostra e padrões.

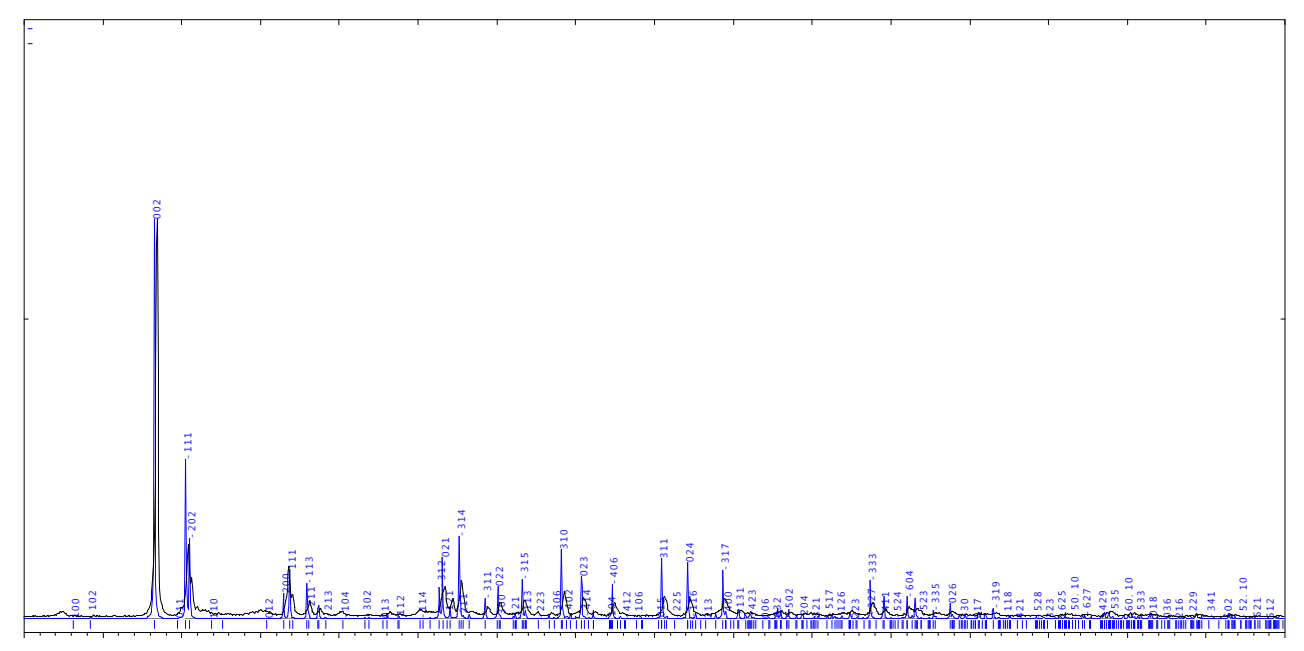

Figura 2 - Sobreposição do difratograma com o padrão simulado da Gibbsita.

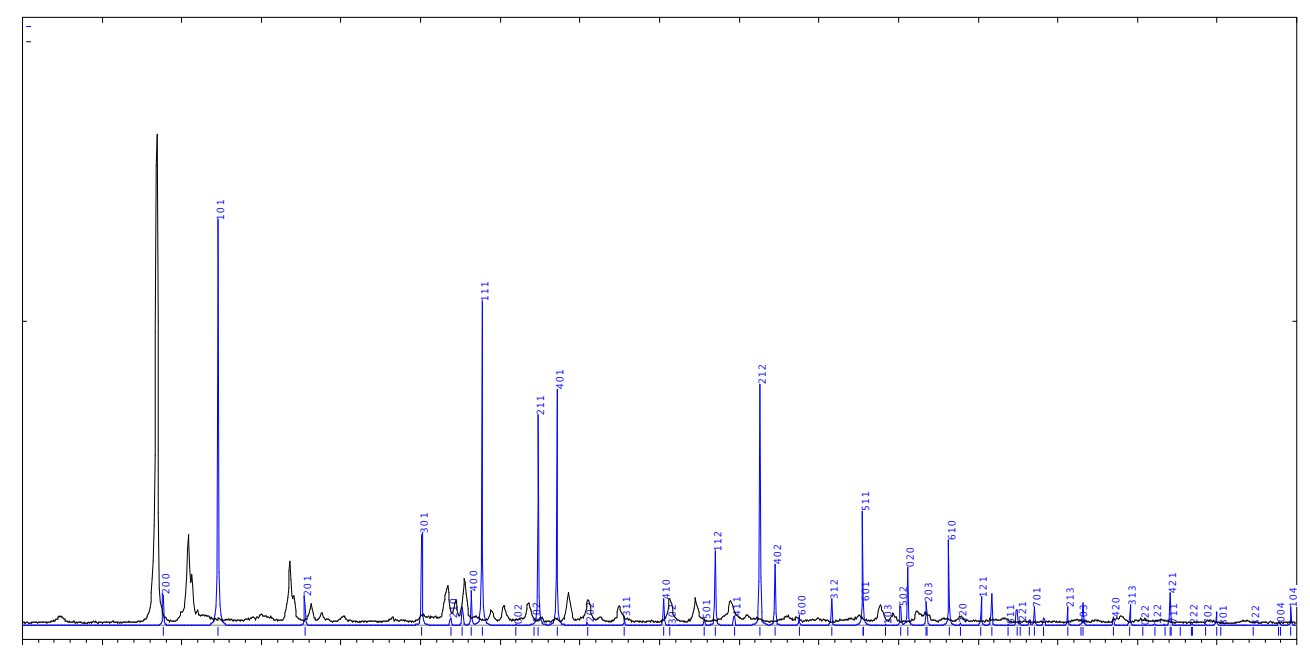

Figura 3 - Sobreposição do difratograma com o padrão simulado do Diásporo. 


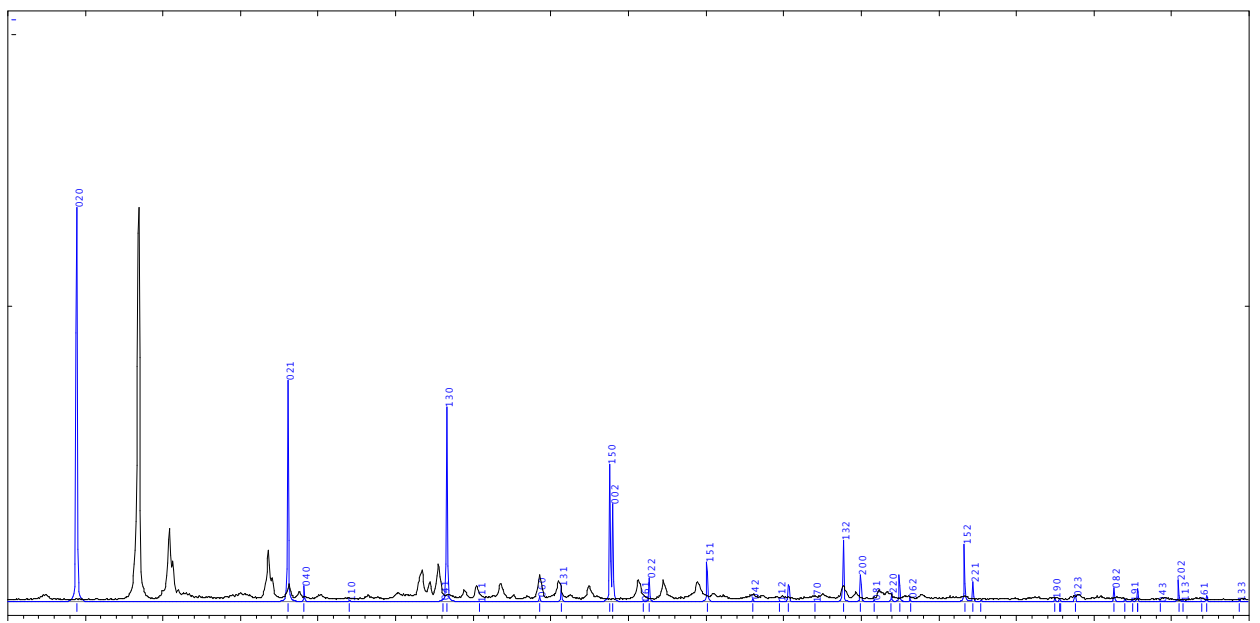

Figura 4 - Sobreposição do difratograma com o padrão simulado da Boehmita.

As Figuras 5 e 6 apresentam os padrões das fases Hematita e magnetita onde também não são observadas sobreposições de picos com a amostra.

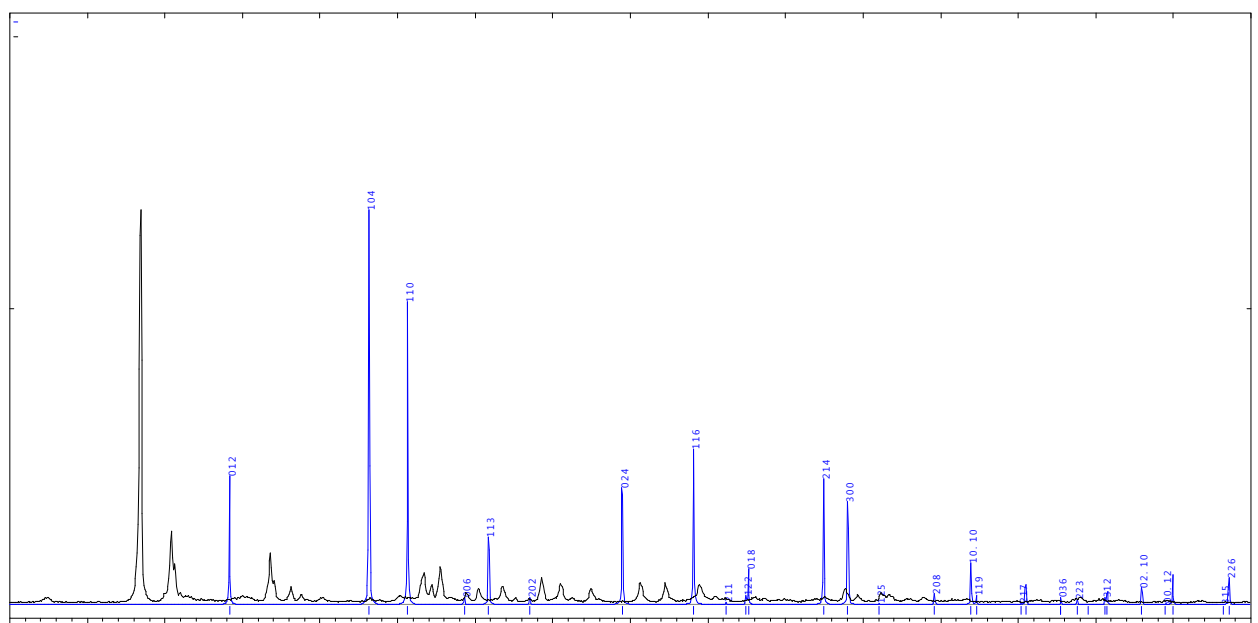

Figura 5 - Sobreposição do difratograma com o padrão simulado da Hematita. 


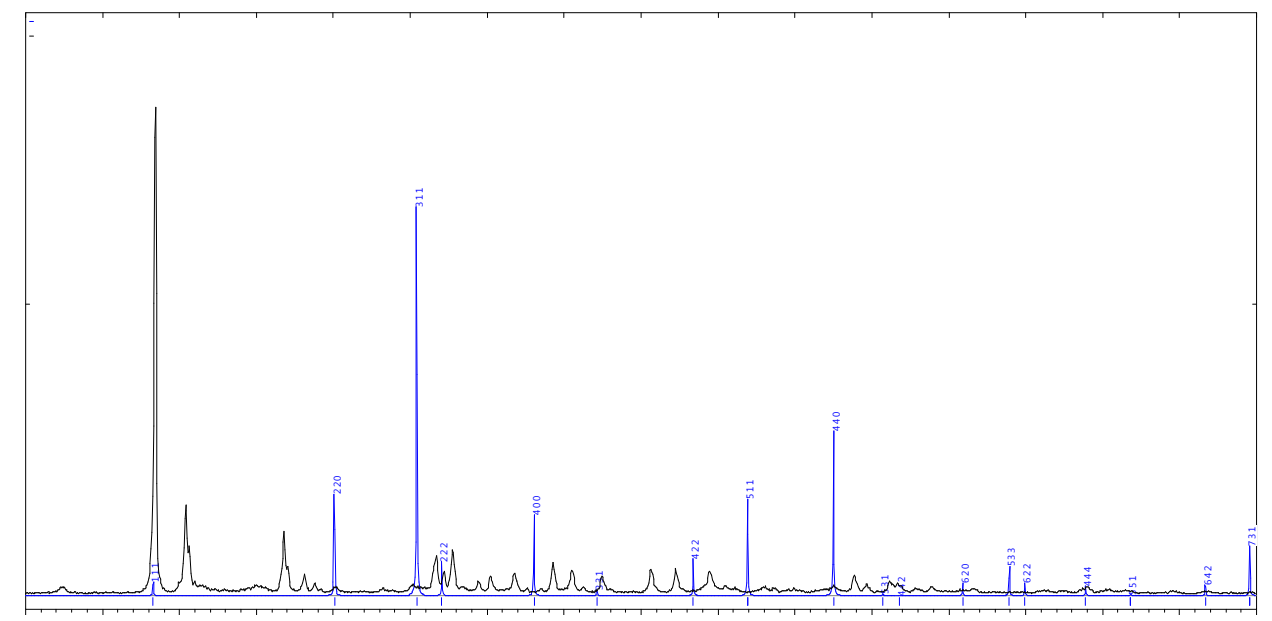

Figura 6 - Sobreposição do difratograma com o padrão simulado da Magnetita.

A Figura 7 apresenta a sobreposição da Caulinita no qual observa-se a sobreposição do pico de mais alta intensidade do padrão da Caulinita com um pico de baixa intensidade da amostra posicionado próximo a $2 \theta=12,5^{\circ}$ indicando que esta fase deve estar presente em pequena quantidade nesta amostra.

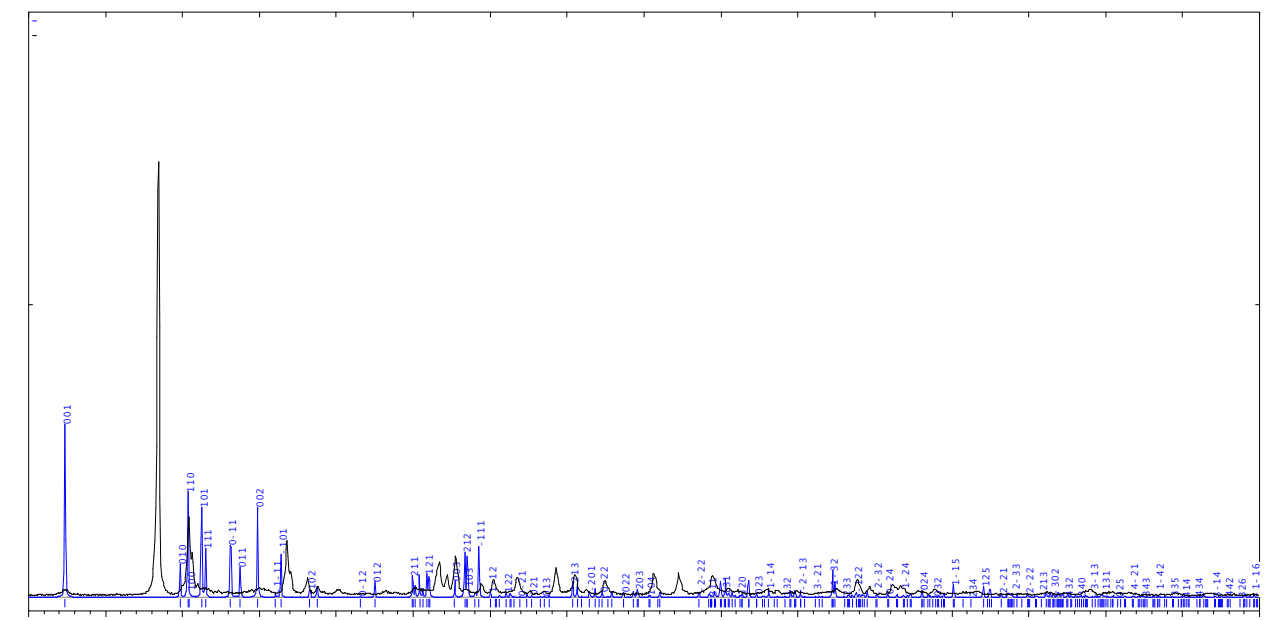

Figura 7 - Sobreposição do difratograma com o padrão simulado da Caulinita.

As Figuras 8, 9 e 10 apresentam os padrões das fases Quartzo, Rutilo e Anatásio, nas quais não são observados a sobreposição dos picos da amostra e padrões. 


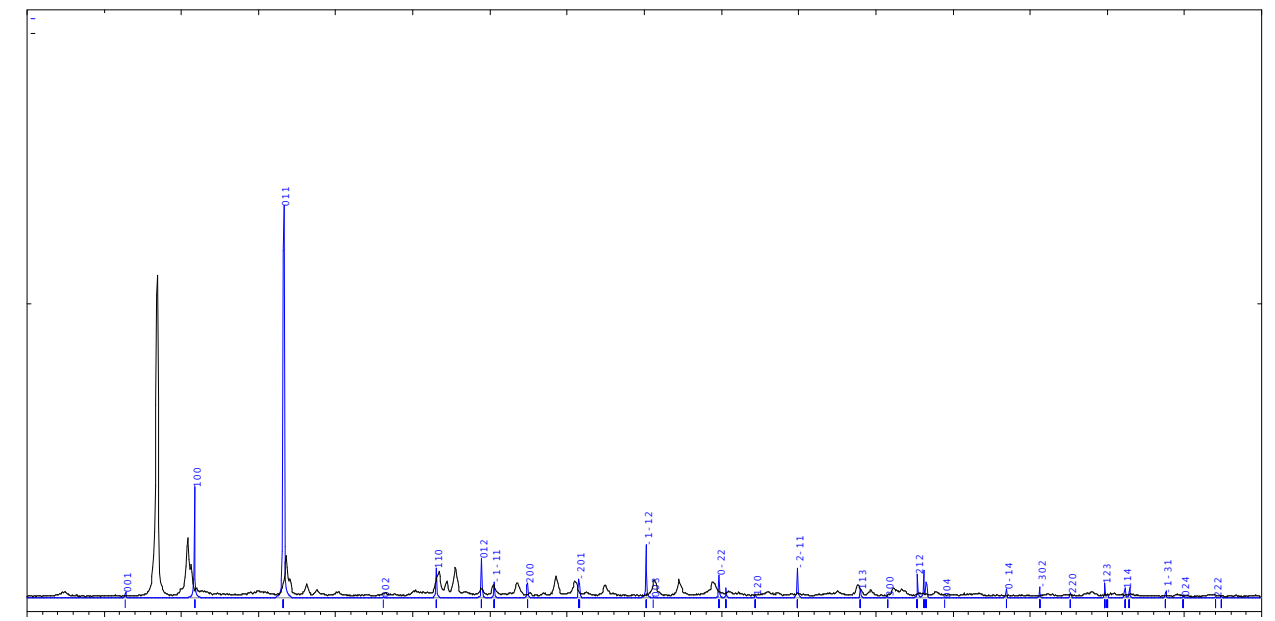

Figura 8 - Sobreposição do difratograma com o padrão simulado do Quartzo.

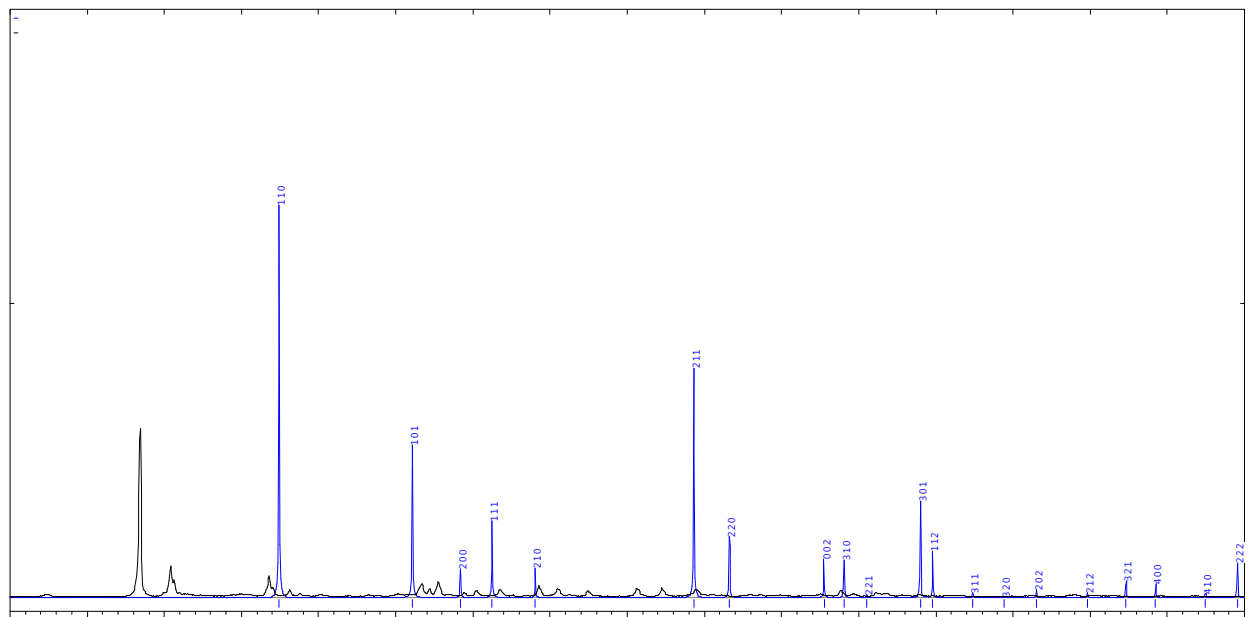

Figura 9 - Sobreposição do difratograma com o padrão simulado do Rutilo. 


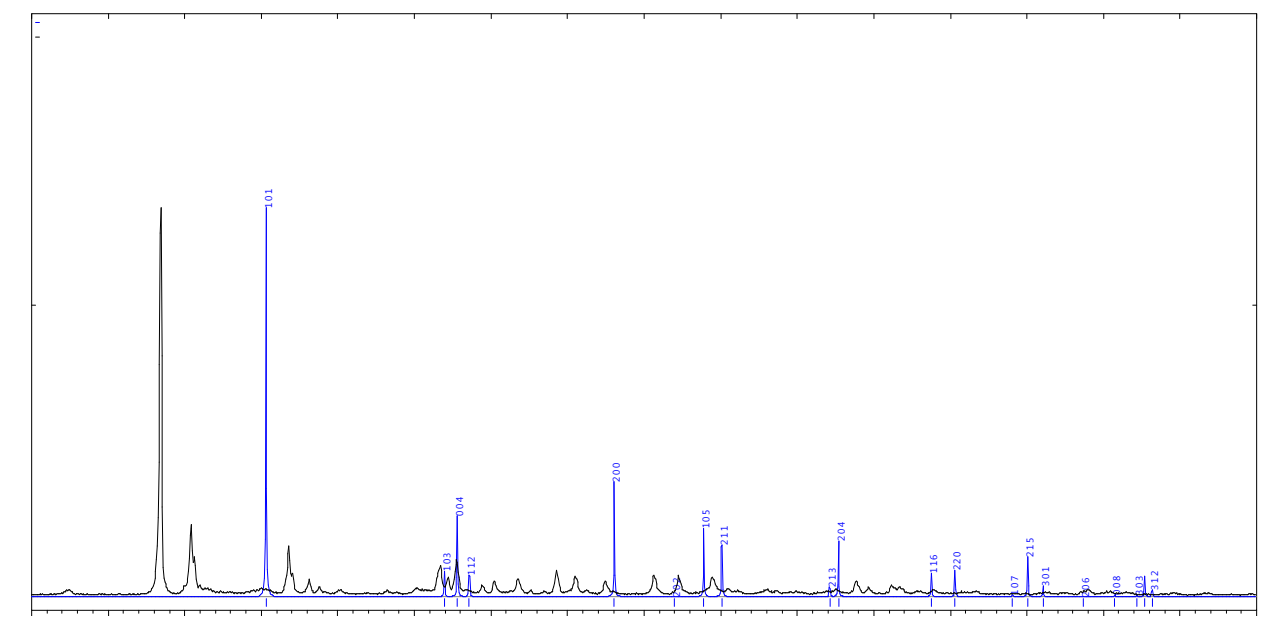

Figura 10 - Sobreposição do difratograma com o padrão simulado do Anatásio.

Estes resultados indicam que a amostra analisada é constituída majoritariamente pela fase Gibbisita.

\section{CONCLUSÕES}

Este trabalho mostra que a Bauxita, extraída da região de Lavrinhas/SP utilizada na fabricação do coagulante Sulfato de Alumínio, caracterizada microestruturalmente por DRX é composta majoritariamente pela fase Gibbsita.

\section{REFERÊNCIAS}

EVANS, K. A. Industrial Inorganic Chemicals: Production and Uses; ed. Cambridge:The Royal Society of Chemistry, 1995.

GREENWOOD, N. N.; EARNSHAW, A. Chemistry of the Elements, ed. Oxford: Butterworth Heinemann, 1997.

KRAUS W.; NOLZE G. PowderCell (2.3). Berlim: Fed. I. Res. Test, 1999.

MEGAW, H. D. The crystal structure of hydrargillite, $\mathrm{Al}(\mathrm{OH})_{3}$. Zeitschrift für Kristallographie - Crystalline Materials, Cambridge, v. 87, p. 185-204, 1934.

PAULING, L. The structure of the micas and related minerals. Proceedings of the National Academy of Sciences of the United States of America, Washington, v. 16, p. 123-129, jan. 1930 .

PEARSON P.; CALVERT L. D. Pearson's Handbook of Crystallographics data for Intermetallic Phases. 2nd. Ed. Metals park: ASM international, 1991.

SAALFELD, H.; WEDDE, M. Refinement of the crystal structure of gibbsite, $\mathbf{A l}(\mathbf{O H})_{3}$. Zeitschrift für Kristallographie, v. 139, p. 129-135, 1974.

U.S. GEOLOGICAL SURVEY. Mineral Commodity Summaries 2017, Reston, Virginia, 2017. Disponível em: <https://minerals.usgs.gov/minerals/pubs/mcs $/ 2017 / \mathrm{mcs} 2017$.pdf $>$. Acesso em: 03 julho de 2017. 